\title{
Risk Factors and Timing of Native Kidney Biopsy Complications
}

\author{
Marie-Christine Simard-Meilleur ${ }^{a}$ Stéphan Troyanov ${ }^{c} \quad$ Louise Roy $^{a}$ \\ Etienne Dalaire $^{b}$ Soumeya Brachemi ${ }^{a}$ \\ Divisions of a Nephrology and b Radiology, Centre Hospitalier de I'Université de Montréal, \\ Hôpital Saint-Luc, and 'Nephrology Division, Hôpital du Sacré-Cœur de Montréal, \\ Montreal, Que., Canada
}

\section{Key Words}

Kidney biopsy $\cdot$ Timing of complications $\cdot$ Hematoma $\cdot$ Risk factors

\section{Abstract}

Background: The appropriate observation period, rate and risk factors of complications after a percutaneous renal biopsy remain debated. Methods: We retrospectively studied native kidney biopsies performed in our institution between January 2007 and July 2011. Outpatients had either an 8- (67\%) or a 24-hour (33\%) observation period. Results: 312 biopsies were reviewed (287 patients), $51 \%$ of patients were female and the mean age was $54 \pm 15$ years. Half of these biopsies were performed in outpatients. A total of $15 \%$ of patients developed a symptomatic hematoma, $9 \%$ received a red blood cell transfusion and $1 \%$ required an angio-intervention. Eighty-four percent of the complications manifested within the first $8 \mathrm{~h}, 86 \%$ at $12 \mathrm{~h}$ and $94 \%$ at $24 \mathrm{~h}$. Outpatients experienced significantly less complications, all manifesting within the first $8 \mathrm{~h}, 14 \%$ required an observation period longer than planned. The risk of symptomatic hematoma increased to $11,20,35$ and $40 \%$ in patients with $>200,140-200,100-140$ and $<100 \times 10^{9} / /$ platelets, respectively $(p=0.002)$. It also increased in hemodialysis patients ( $29 \%$ compared to $14 \%, p=0.02$ ). We found no association of risk with the number of biopsy passes and only a trend with needle size. Conclusion: Symptomatic hematomas occurred in $15 \%$ of kidney biopsies and were strongly associated with platelet count and hemodialysis. Outpatients experienced fewer complications; therefore, we can conclude that same-day discharge in selected patients is safe. 


\section{Introduction}

Native kidney biopsy is a useful tool to establish a diagnosis, treatment and prognosis of glomerular and tubulointerstitial diseases. It reveals a diagnosis different than anticipated in $50 \%$ of cases, and its result modifies treatment in $20-50 \%$ of cases [1]. Yet, because of bleeding complications [2-4], physicians are at times reluctant to request a native kidney biopsy [5-7]. Events related to the procedure usually occur within the first $24 \mathrm{~h}$. Some studies have concluded that the majority manifest within $8 \mathrm{~h}$ [1], while others maintain that a short observation time misses more than a third of complications and recommend a 24-hour waiting period [8]. Whether a selected group of patients can be safely discharged the same day following a kidney biopsy is still debated [9-14]. Doing so, while insuring patient safety, using a valid risk assessment would positively impact their quality of life and also reduce health care costs.

This study addressed the rate and risk factors of symptomatic hematomas following native kidney biopsy. We also evaluated if same-day discharge is safe.

\section{Patients and Methods}

\section{Design}

This was a retrospective study designed for an internal quality project to evaluate renal biopsies at our institution. It was approved by our institutional review board, and as so, was exempted from our ethics committee approval. All native kidney biopsies performed the Centre Hospitalier de l'Université de Montréal (CHUM), Montreal, Que., Canada, between January 2007 and July 2011 were included.

\section{Procedure}

Native kidney biopsies were performed by radiologists using an automated or semi-automated needle under ultrasound or CT scan guidance. The procedure was usually done at the lower pole of the kidney unless performed for investigation of renal mass. Patients stopped anticoagulation therapy appropriately and antiplatelets use at least 7 days prior to biopsy. All patients had a complete blood count, international normalized ratio (INR) and partial thromboplastin time (PTT) prior to the procedure. Platelet (PLT) function tests (PFA-100 test or bleeding time) were not systematically performed. After the biopsy, the patients maintained bed rest for $6 \mathrm{~h}$. Vital signs and urine aspect were checked regularly. Postbiopsy imaging was done when clinically indicated at the discretion of the attending physician or following a location protocol.

\section{Data Collection}

Information reviewed from charts included demographic data, history of liver disease, type of renal presentation, blood pressure within the week prior to biopsy, serum creatinine, eGFR (using the MDRD formula), hemoglobin (Hb), PLT, INR, PTT and bleeding time or PFA-100. We obtained information on kidney size, needle size, number of needle passes and number of glomeruli obtained. We recorded the administration of fresh frozen plasma (FFP), PLTs and red blood cell (RBC) transfusions as well as the use of desmopressin.

\section{Definitions}

Uncontrolled hypertension was defined as $>50 \%$ of systolic and diastolic readings of $>140 />90 \mathrm{~mm} \mathrm{Hg}$ within the week prior to biopsy. We noted the occurrence and timing of symptomatic hematoma (hematoma with abdominal pain warranting radiologic assessment, 
Table 1. Biopsy procedure and adequacy $(\mathrm{n}=312)$

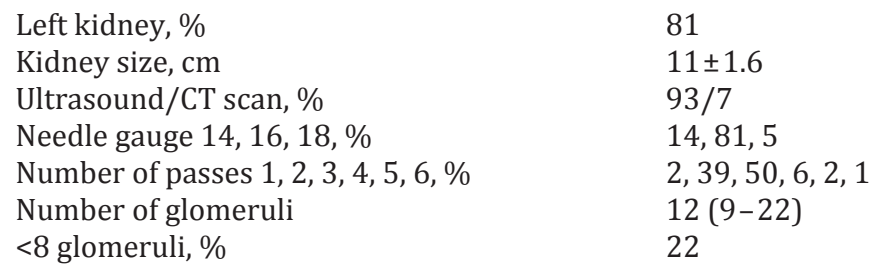

Values are expressed as mean \pm standard deviation or median (interquartile range), except where otherwise indicated. A minimum of 8 glomeruli was defined as an adequate specimen according to the Banff classification of renal allograft pathology.

hypotension, a drop in $\mathrm{Hb}$ of $\geq 10 \mathrm{~g} / \mathrm{l}$ or macroscopic hematuria), urinary infection, the need for $\mathrm{RBC}$ transfusion or angio-intervention. Inpatients are defined by a preexisting hospitalization prior to biopsy. According to local practice, outpatients were observed for either 24 or $8 \mathrm{~h}$.

\section{Statistical Analysis}

We analyzed risk factors of symptomatic hematoma and compared inpatients to 8- and 24-hour outpatients. Normally distributed variables are expressed as mean \pm standard deviation and compared using t test or one-way ANOVA. Non-normally distributed variables are expressed as median with interquartiles (Q1-Q3) and compared using Mann-Whitney U test or Kruskal-Wallis test. Categorical data are compared using $\chi^{2}$ test. All $\mathrm{p}$ values were 2 -tailed, and values $<0.05$ were considered statistically significant. Analyses were carried out using SPSS software (version 20; IBM, North Castle, N.Y., USA).

\section{Results}

\section{Cohort Characteristics}

A total of 312 native kidney biopsies were performed in 287 patients. The mean age was $54 \pm 15$ years, $51 \%$ of patients were females, median eGFR was $37(18-74) \mathrm{ml} / \mathrm{min} / 1.73 \mathrm{~m}^{2}$, $10 \%$ were on dialysis, $8 \%$ had liver disease and $17 \%$ had uncontrolled hypertension. Fiftythree percent of biopsies were done in inpatients, 16 and 31\% in outpatients scheduled for a 24- or 8-hour observation period, respectively. Information was missing in $<5 \%$ of cases for all variables except for PLT function tests, drop in $\mathrm{Hb}$ and kidney size, missing in 60, 17 and $42 \%$ of cases, respectively. Imaging was in fact available in $84 \%$ of patients prior to biopsy, but kidney size was not always specified, other than presence or absence of atrophy.

Seven percent of biopsies were performed for the diagnostic workup of renal masses, $19 \%$ for nephrotic range proteinuria, $9 \%$ for rapidly progressive renal insufficiency, $18 \%$ for acute kidney injury, $11 \%$ for a chronic kidney disease, and the remaining $36 \%$ for nonnephrotic proteinuria and/or hematuria. Most biopsies were done under ultrasound guidance using a 16-gauge needle with three needle passes (table 1). Eleven percent of the available bleeding times were elevated. Overall, 33\% of patients received desmopressin, 4\% PLTs, and/or $4 \%$ FFP.

\section{Risk Factors of Complications}

The incidence of symptomatic hematoma was 15\%, 9\% of patients needed RBC transfusion and 3 inpatients required an angio-intervention (table 2). Individuals requiring RBC 
Table 2. Complications

Simard-Meilleur et al.: Risk Factors and Timing of Native Kidney Biopsy Complications

$\begin{array}{lr}\text { Symptomatic hematoma, \% } & 15 \\ \text { Pain warranting radiologic assessment, \% } & 7 \\ \text { Drop of } \geq 10 \text { g/l in Hb, \% } & 13 \\ \text { Macroscopic hematuria*, \% } & 2 \\ \text { Symptomatic hypotension, \% } & 3 \\ \text { Macroscopic hematuria, \% } & 5 \\ \text { RBC transfusion, \% } & 9 \\ \text { Angio-intervention, \% } & 1 \\ \text { Urinary infection, n } & 1\end{array}$

* With a radiological hematoma. Categories are not mutually exclusive.

Table 3. Timing of complications

\begin{tabular}{lclcr}
\hline & $<8 \mathrm{~h}$ & $8-12 \mathrm{~h}$ & $12-24 \mathrm{~h}$ & $\geq 24 \mathrm{~h}$ \\
\hline All patients, \% & 84 & 2 & 8 & 6 \\
Inpatients, \% & 72 & 3 & 15 & 10 \\
Outpatients, \% & 100 & 0 & 0 & 0 \\
\hline
\end{tabular}

transfusion had an initial $\mathrm{Hb}$ of $92 \pm 14 \mathrm{~g} / \mathrm{l}$ as opposed to $115 \pm 22 \mathrm{~g} / \mathrm{l}(\mathrm{p}<0.001)$ in those who did not. Twenty percent of transfused patients had a drop in $\mathrm{Hb}$ of $<10 \mathrm{~g} / \mathrm{l}$. Among the transfused patients, only 55\% had postbiopsy imaging, of these $20 \%$ had no hematoma.

There was no nephrectomy or death. At $8 \mathrm{~h}, 84 \%$ of patients who developed complications had clinical manifestations. This increased to $86 \%$ at $12 \mathrm{~h}$ and $94 \%$ at $24 \mathrm{~h}$ (table 3).

The PLT count prior to biopsy was highly predictive of symptomatic hematoma (fig. 1). Among those with a low PLT count $\left(<140 \times 10^{9} / \mathrm{l}, \mathrm{n}=30\right), 7$ received PLT transfusion prior to biopsy and $29 \%$ had a symptomatic hematoma as opposed to $39 \%$ in those without a PLT transfusion $(\mathrm{p}=0.61)$. Although eGFR was not predictive of symptomatic hematoma, individuals receiving hemodialysis were more likely to bleed ( 29 vs. $14 \%, p=0.02$ ). Finally, outpatients experienced fewer events (table 4).

We found no statistical association between bleeding complications and age, sex, uncontrolled hypertension, hepatic disease, biopsy by ultrasound or CT scan, Hb, INR, PTT or abnormal PLT tests. The use of desmopressin and FFP did not translate into fewer complications although patients who received these treatments had lower PLTs and eGFR, and a higher INR (data not shown). Interestingly, there was no association with the number of needle passes with a median of three passes in individuals with and without a symptomatic hematoma. There was a trend toward fewer symptomatic hematomas with 18-gauge needles (fig. 2) and statistically more glomeruli when a 16-gauge needle or larger was used (fig. 3).

\section{Inpatients and Outpatients}

Overall, 148 biopsies were performed in outpatients, 99 had an 8-hour observation period and 49 a 24-hour stay. Inpatients differed with regard to comorbidities, eGFR, preventive treatments and complications (table 4). The outpatients group more frequently received desmopressin despite a lower rate of abnormal PLT tests. This paradox is created by the 24-hour hospital protocol in which PLT function tests are routinely measured and desmopressin is proposed. Overall, $27 \%$ of inpatients experienced any complications as opposed to $14 \%$ of outpatients $(\mathrm{p}=0.002)$. 
Fig. 1. Symptomatic hematoma and PLT level. PLT count was missing in 1 subject. The number of individuals within each category who received PLT transfusion is given in parentheses. The normal PLT count was $\geq 140 \times 10^{9} / \mathrm{l}$ in our laboratory.

Table 4. Comparison of inpatients and outpatients
Simard-Meilleur et al.: Risk Factors and Timing of Native Kidney Biopsy Complications

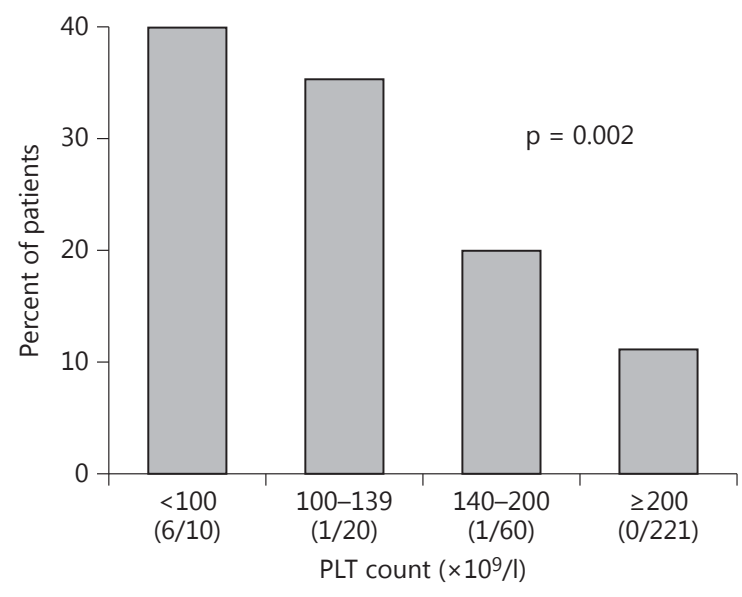

\begin{tabular}{lllc}
\hline & $\begin{array}{l}\text { Inpatients } \\
(53 \%)\end{array}$ & $\begin{array}{l}\text { Outpatients } \\
(47 \%)\end{array}$ & $\mathrm{p}$ \\
& $54 \pm 16$ & $53 \pm 15$ & 0.67 \\
\hline Age, years & 48 & 55 & 0.25 \\
Female gender, \% & 7 & 0.04 \\
Uncontrolled hypertension, \% & 14 & 5 & 0.10 \\
Hepatic disease, \% & 10 & 1 & $<0.001$ \\
Dialysis, \% & 18 & $54(32-83)$ & $<0.001$ \\
eGFR, ml/min/1.73 m ${ }^{2}$ & $24(10-53)$ & $125 \pm 20$ & $<0.001$ \\
Hb, g/l & $101 \pm 18$ & $222 \pm 103$ & 0.002 \\
PLT, $\times 10 \% / l$ & $270 \pm 116$ & $1.0(1.0-1.1)$ & $<0.001$ \\
INR & $1.0(0.9-1.0)$ & 0.95 \\
Abnormal platelet function & 11 & 11 & \\
test, \% & & 0 & 0.006 \\
PLT transfusion, \% & 5 & 1 & 0.01 \\
FFP, \% & 6 & 22 & $<0.001$ \\
Desmopressin, \% & 43 & 12 & 0.13 \\
Symptomatic hematoma, \% & 18 & 4 & 0.55 \\
Macroscopic hematuria, \% & 6 & 0 & 0.10 \\
Angio-intervention, $\mathrm{n}$ & 3 & 1 & $<0.001$ \\
Transfusion, \% & 17 & & \\
\hline
\end{tabular}

Results are expressed as mean \pm standard deviation or median (interquartile range), except where otherwise indicated.

Outpatients: 8- and 24-Hour Observation Period

The decision to observe patients 8 or $24 \mathrm{~h}$ was based on each hospital's protocol. These two subgroups did not differ in eGFR, mean $\mathrm{Hb}$ or coagulation studies prior to biopsy. The incidence of complications was similar in both groups and all happened within the first $8 \mathrm{~h}$ of observation (table 3).

Hospitalization of outpatients after biopsy occurred in 14\% (15 of 99 in the 8-hour and 5 of 49 in the 24-hour subgroups). Among these 20 patients, 11 had a symptomatic hematoma, 5 had a $\geq 10 \mathrm{~g} / \mathrm{l}$ drop in $\mathrm{Hb}$ without an evident hematoma, 2 had macroscopic hematuria, and 3 required RBC transfusion. Only 1 patient observed for $8 \mathrm{~h}$ was discharged as planned but returned to the emergency room for abdominal pain after $24 \mathrm{~h}$; however, no complication was found on imaging. No outpatient was lost during the follow-up after the biopsy. 
Fig. 2. Symptomatic hematoma and size of biopsy needle. Needle biopsy data was missing in 6 subjects. The number of individuals within each category is given in parentheses. The median (interquartile ranges given in parentheses) number of passes is shown.

Fig. 3. Biopsy needle size and number of glomeruli. Needle biopsy data was missing in 6 subjects, 23 biopsies for renal masses were excluded. The number of individuals within each category is given in parentheses. The median (interquartile ranges given in parentheses) number of passes is shown.
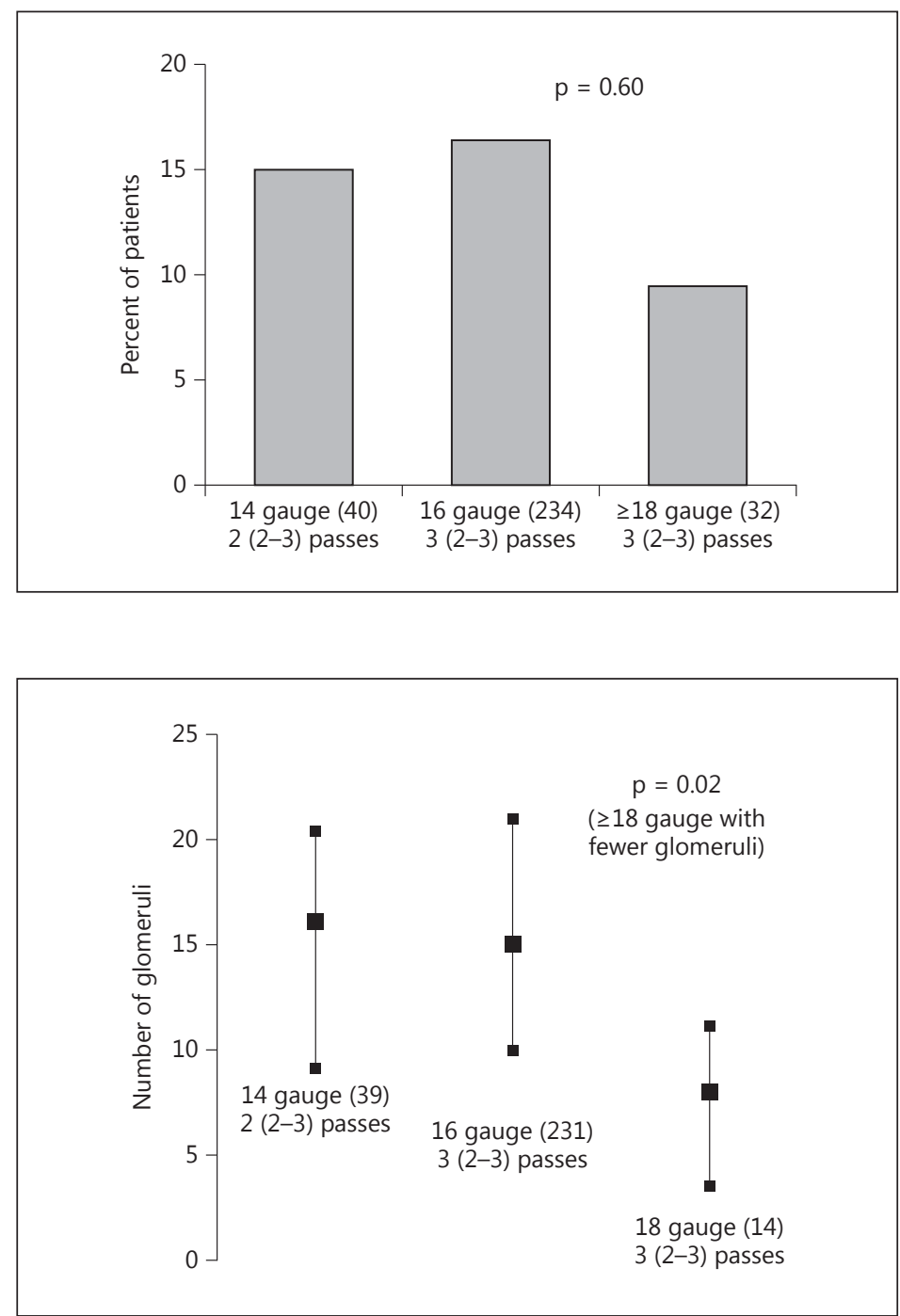

\section{Discussion}

This is a cohort of 312 kidney biopsies of inpatients (53\%) and outpatients (47\%) in our institution. A total of $15 \%$ of patients developed a symptomatic hematoma. Hematoma was not regarded as significant unless it was associated with a clinical complication (abdominal pain warranting radiologic assessment, hypotension, a drop in $\mathrm{Hb}$ of $\geq 10 \mathrm{~g} / \mathrm{l}$ or macroscopic hematuria). Radiologic hematomas without any repercussion were not included for several reasons. Silent perinephric hematomas are less clinically relevant since they can occur in $>85 \%$ of patients after percutaneous biopsy and they are not predictive of major complications $[15,16]$. Moreover, not all patients had a systematic routine ultrasound examination postbiopsy.

It must be noted that arteriovenous fistulas were not reported because they were not systematically looked for. In fact, according to the previous literature these are common but rarely have a clinical impact $[17,18]$.

We found that we had a higher rate of symptomatic hematomas (15\%) compared to the literature (7.8\%) [18]. This is probably due to our definition of symptomatic hematoma that 
includes several clinical events. Another reason may be the inclusion of a large proportion of hospitalized patients. Indeed, there were more symptomatic hematomas in inpatients $(18 \%)$ compared to outpatients (12\%), although it was not statistically significant. The inpatient population had more comorbidities including dialysis (18 vs. 1\%), a lower $\mathrm{Hb}$ at baseline (101 \pm 18 vs. $125 \pm 20$ ) and more PLT transfusion ( $5 \%$ vs. none).

A higher transfusion rate was also found when compared to previously reported studies ( 9 vs. $0.4-6 \%$ ) [19-22]. Indeed, a large number of patients had a low $\mathrm{Hb}$ concentration prior to biopsy as a mild decrease in $\mathrm{Hb}$ can lead to transfusion and a low $\mathrm{Hb}$ itself might be a risk factor for bleeding [11]. However, of the transfused patients who had a postbiopsy imaging, $20 \%$ did not have a hematoma. The decrease in $\mathrm{Hb}$ without the finding of a radiologic hematoma can be explained by hemodilution from a saline infusion before and after the biopsy. It can also depend on the modality of imaging, since CT scans are more sensitive in detecting hematoma than echography.

We found that the PLT level was a statistically significant risk factor for symptomatic hematoma. There was also a non-significant trend for less symptomatic hematomas when patients with thrombocytopenia received PLT transfusion prior to biopsy. We did not find that age, uncontrolled hypertension, and a decreased GFR were associated with an increased risk of hematoma or blood transfusion $[11,19]$. Major events did not correlate with neither the needle size nor the number of passes as found in the study by Tondel et al. [23], although there was a non-significant trend for more hematomas with larger needles.

In our whole cohort, $16 \%$ of complications appeared after the first $8 \mathrm{~h}$. Outpatients had fewer complications and these manifested within $8 \mathrm{~h}$, whether they were observed for 8 or $24 \mathrm{~h}$, and $14 \%$ were hospitalized for a longer period than planned. Only 1 outpatient returned to the emergency room (with unrelated abdominal pain), and no further complications were noted on follow-up notes in the other patients' charts.

These results are in line with Lin et al. [1] who found that there is no difference in the rate of complications between patients who are admitted and those observed for a 6-hour period, the latest being acceptable. By contrast, Whittier and Korbet [8] found that $42 \%$ of complications following native kidney biopsy manifested at $\leq 4 \mathrm{~h}, 67 \%$ at $\leq 8 \mathrm{~h}, 85 \%$ at $\leq 12 \mathrm{~h}$, and $89 \%$ at $\leq 24 \mathrm{~h}$. According to this study, the optimal observation period should therefore be $24 \mathrm{~h}$.

Our study has limitations, it is a retrospective study and our population is not homogenous but still represents our daily practice. By including only clinically significant events in the definition of clinical hematoma our goal was to include only events that had a real impact on the patients.

In conclusion, in our study symptomatic hematomas occurred in 15\% of kidney biopsies and were strongly associated with PLT count and hemodialysis. Patients chosen for a biopsy on an outpatient basis had less comorbidities than those who were hospitalized, and they experienced fewer complications all observable within the first $8 \mathrm{~h}$. Therefore, same-day discharge with an 8-hour observation period is a medically adequate procedure in carefully selected patients, a significant finding, since outpatient biopsies are economically advantageous.

\section{Disclosure Statement}

The authors have no competing financial interests to disclose. 


\section{References}

1 Lin WC, Yang Y, Wen YK, Chang CC: Outpatient versus inpatient renal biopsy: a retrospective study. Clin Nephrol 2006;66:17-24.

-2 Corapi KM, Chen JL, Balk EM, Gordon CE: Bleeding complications of native kidney biopsy: a systematic review and meta-analysis. Am J Kidney Dis 2012;60:62-73.

-3 Diaz-Buxo JA, Donadio JV Jr: Complications of percutaneous renal biopsy: an analysis of 1,000 consecutive biopsies. Clin Nephrol 1975;4:223-227.

4 Whittier WL: Complications of the percutaneous kidney biopsy. Adv Chronic Kidney Dis 2012;19:179-187.

5 Mendelssohn DC, Cole EH: Outcomes of percutaneous kidney biopsy, including those of solitary native kidneys. Am J Kidney Dis 1995;26:580-585.

6 Moutzouris DA, Herlitz L, Appel GB, Markowitz GS, Freudenthal B, Radhakrishnan J, D’Agati VD: Renal biopsy in the very elderly. Clin J Am Soc Nephrol 2009;4:1073-1082.

7 Parrish AE: Complications of percutaneous renal biopsy: a review of 37 years' experience. Clin Nephrol 1992; 38:135-141.

8 Whittier WL, Korbet SM: Timing of complications in percutaneous renal biopsy. J Am Soc Nephrol 2004;15: 142-147.

- Fraser IR, Fairley KF: Renal biopsy as an outpatient procedure. Am J Kidney Dis 1995;25:876-878.

10 Jones B, Puvaneswary M, Nanra R, Trevillian P, Carney S, Gillies A: Reduced duration of bed rest after percutaneous renal biopsy. Clin Nephrol 1991;35:44-45.

11 Marwah DS, Korbet SM: Timing of complications in percutaneous renal biopsy: what is the optimal period of observation? Am J Kidney Dis 1996;28:47-52.

-12 McMahon GM, McGovern ME, Bijol V, Benson CB, Foley R, Munkley K, Schnipper J, Franz C, Lin J: Development of an outpatient native kidney biopsy service in low-risk patients: a multidisciplinary approach. Am J Nephrol 2012;35:321-326.

13 Murphy BF, MacIsaac A: Percutaneous renal biopsy as a day-patient procedure. Am J Kidney Dis 1989;14:77.

14 Simckes AM, Blowey DL, Gyves KM, Alon US: Success and safety of same-day kidney biopsy in children and adolescents. Pediatr Nephrol 2000;14:946-952.

-15 Ishikawa E, Nomura S, Hamaguchi T, Obe T, Inoue-Kiyohara M, Oosugi K, Katayama K, Ito M: Ultrasonography as a predictor of overt bleeding after renal biopsy. Clin Exp Nephrol 2009;13:325-331.

-16 Waldo B, Korbet SM, Freimanis MG, Lewis EJ: The value of post-biopsy ultrasound in predicting complications after percutaneous renal biopsy of native kidneys. Nephrol Dial Transplant 2009;24:2433-2439.

17 Madaio MP: Renal biopsy. Kidney Int 1990;38:529-543.

18 Stratta P, Canavese C, Marengo M, Mesiano P, Besso L, Quaglia M, Bergamo D, Monga G, Mazzucco G, Ciccone G: Risk management of renal biopsy: 1387 cases over 30 years in a single centre. Eur J Clin Invest 2007;37: 954-963.

19 Hergesell O, Felten H, Andrassy K, Kuhn K, Ritz E: Safety of ultrasound-guided percutaneous renal biopsyretrospective analysis of 1,090 consecutive cases. Nephrol Dial Transplant 1998;13:975-977.

20 Mackinnon B, Fraser E, Simpson K, Fox JG, Geddes C: Is it necessary to stop antiplatelet agents before a native renal biopsy? Nephrol Dial Transplant 2008;23:3566-3570.

21 Whittier WL, Korbet SM: Renal biopsy: update. Curr Opin Nephrol Hypertens 2004;13:661-665.

-22 Torres Munoz A, Valdez-Ortiz R, Gonzalez-Parra C, Espinoza-Davila E, Morales-Buenrostro LE, Correa-Rotter R: Percutaneous renal biopsy of native kidneys: Efficiency, safety and risk factors associated with major complications. Arch Med Sci 2011;7:823-831.

23 Tondel C, Vikse BE, Bostad L, Svarstad E: Safety and complications of percutaneous kidney biopsies in 715 children and 8,573 adults in Norway 1988-2010. Clin J Am Soc Nephrol 2012;7:1591-1597. 